\title{
A Network-Based Approach for Modeling Resonant Capacitive Wireless Power Transfer Systems
}

\author{
Eli Abramov, Ilya Zeltser, and Mor Mordechai Peretz
}

\begin{abstract}
In this paper, a network-based approach to model capacitive wireless power transfer systems is introduced. The modeling methodology provides insights into the electrical crosscoupling relationships between input and output parameters of the capacitive power transfer (CPT) systems, including the effect of distance and alignment of the coupling plates. It is revealed that, regardless of the circuit complexity or matching network order, the model core can be reduced to a basic gyrator relationship with added coefficients when required, thus obtaining a compact, closed-form relationship between the input and output terminals. The model has been validated through rigorous simulations and experiments; all found to be in excellent agreement with the theoretical predictions under changes of the air-gap, and medium capacitance. To this end, an experimental CPT prototype that operates in the $\mathrm{MHz}$ range has been designed and implemented while the transmitter and receiver have been realized by four $170 \mathrm{~mm} \times 170 \mathrm{~mm}$ copper plates. In addition, to provide better insight into the capacitive interface under different structures and distances and alignments, the capacitive coupler has been methodically examined through Finite Elements Analysis (FEA) tools Maxwell (Ansys). The results of the FEA have been utilized in the simulation platform to enhance the accuracy of the simulations, accounting for the variable capacitance under variations.
\end{abstract}

Index Terms-Behavioral modeling, capacitive power transfer, capacitive coupling, gyrator, matching networks, two-port network.

\section{INTRODUCTION}

$\mathrm{O}$ VER the last few years, capacitive power transfer (CPT) is a rapidly growing technology in the field of wireless power transfer (WPT) [1]-[7]. One of the more attractive advantages of capacitive-based WPT is the avoidance of undesired Eddy currents and electromagnetic interfaces (EMI) that comes with magnetic based WPT methods [8]-[10]. In addition to efficiency improvements, CPT systems are potentially with lower volume and construction complexity [1]-[7]. However, the power transfer capability and efficiency still depend on the distance and alignment between the transmitting and receiving sides, which is an inherent feature of near-field WPT systems [11], [12].

Manuscript received January 25, 2019. This work was supported by the Prof. A. Pazi Research Foundation. This paper was presented in part at the 2018 IEEE International Power Electronics and Application Conference and Exposition (PEAC), Shenzhen, China, November 2018.

E. Abramov and M. M. Peretz are with the Center for Power Electronics and Mixed-Signal IC, Department of Electrical and Computer Engineering, BenGurion University of the Negev, Beer-Sheva 8410501, Israel (e-mail: eliab@ post.bgu.ac.il; morp@bgu.ac.il).

I. Zeltser is with Power Electronics Department, Rafael Advanced Defense Systems Ltd, P. O. Box 2250, Haifa 31021, Israel (e-mail: ilyaz@rafael.co.il).

Digital Object Identifier 10.24295/CPSSTPEA.2019.00003

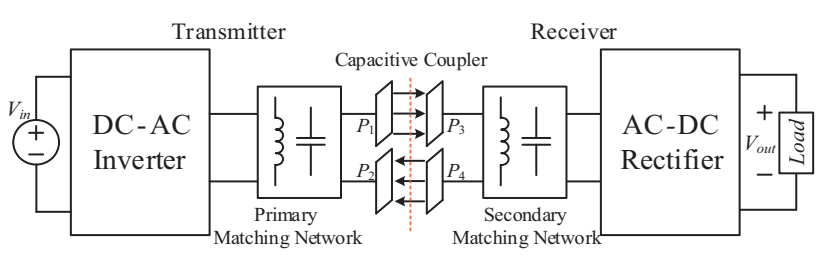

Fig. 1. Simplified diagram of capacitive WPT system.

Several explorations and remedies for extended range power transfer are covered in the literature [13]-[16], for general and capacitive power transfer. An important step in the development of both uniform and specific solutions for extended range WPT systems is a generalized description of the energy transfer mechanism. This requires description of the transfer medium, circuit behavior and their interaction. In particular, in case that active compensation is added, a description of the system's dynamic response is essential. On the topic of the magnetic field based WPT, there can be found several through circuit and system analyses, however, a generic behavioral model and modeling methodology for CPT has not been addressed to-date.

A simplified block diagram of a descriptive CPT system is shown in Fig. 1. In a similar way to magnetic field approaches, reactive networks on both the primary and secondary sides are used for impedance matching between the source and load characteristics [14]-[16]. To achieve degrees of freedom in terms of design, performance and overall input-output relationships in any WPT system, high-order matching networks are used [3], [16], [17]. Analysis of such high-order networks can be quite complex and tedious. As a result, the intricate interaction between the system parameters and characteristics may be overlooked or even missed. Several approaches have been employed to decipher the operation of high-order resonant structures such as analytical multivariable matrices manipulations, geometrical representations [18], [19], and averaging [20], [21]. Circuit derivation of CPT by superpositioning theorem [3], [5], [7], [22], provides clear closedform expressions to the power delivery and other important relationships. However, since some approximations are involved, accurate overall description not always obtained, in particular for a description of the dynamic characteristics. Numerical simulations are also a strong tool to evaluate and characterize different resonant circuits, this approach, however, losses generality and may be time-consuming for cases that a time domain cycle-by-cycle simulation is carried out [23]-[25]. It would be extremely beneficial if a simple and unified generic 
averaging behavioral modeling methodology for capacitivebased WPT systems is utilized.

The objective of this study is therefore to introduce a network-based approach to describe the behavior of capacitive WPT system, under variations of the source and the load circuits, coupling interface and matching networks. The modeling methodology results in a unified model for CPT that provides an insight to the cross-coupling relationship between the input and output parameters and enables to account for changes in distance and alignment of the coupling plates.

The rest of the paper is organized as follows: Following a brief survey of WPT matching structures, Section II details twoport network-based analysis. Section III delineates a case study of a network-based approach to analyze a CPT system. Model validation through various simulations including continuous capacitive medium variations is provided in Section IV. Experimental results of a capacitive WPT prototype are delineated in Section V. Section VI concludes the paper.

\section{Review of Two-Port Representation of Matching NETWORKS}

\section{A. Matching Network Configurations}

Fig. 2 shows several popular matching networks that are mostly used in capacitive WPT systems [1]-[7]. A well-known and simple option is depicted in Fig. 2(a) in which a series inductance is connected to the coupling capacitance of the wireless medium forming a resonator [2]. By this, operation in the vicinity of the resonant frequency results in efficient power transfer to the load. However, a significant drawback of this configuration is that any change either in the capacitive medium or the load parameters, even the slightest, results in different operating point and a significant deterioration of the system ability to transfer power. This is somewhat alleviated by the configuration in Fig. 2(b), in which both the primary and secondary sides of the coupling element include series-parallel resonators (Double-Sided $L C$ ). Here, the operating frequency is near the matching network's resonance, and therefore the system is less sensitive variations in the coupling capacitance (due to distance or misalignment variations). At the cost of slightly higher component count, the power transfer capabilities are higher than series compensation, but still depends on the coupling for efficiency and delivered power [5], [7].The structure in Fig. 2(c) is a combination of the $L C$ matching network with an additional series inductor on both sides of the system (Double-Sided $L C L$ ). By proper design of the inductors $\left(L_{P}, L_{P 1}, L_{S}, L_{S 1}\right)$, this setup allows several degrees of freedom to adjust the power transfer of the system. However, as in the case of $L C$ matching network, the power transfer capabilities of $L C L$ structure is inversely proportional to the coupling capacitance [2], [4]. A more complex matching network is shown in Fig. 2(d). It consists of a larger number of reactive elements on both primary and secondary (Double-Sided $L C L C$ ). There, the coupling capacitance in this case does not directly influence the resonance of the matching networks, it is, however, still limits

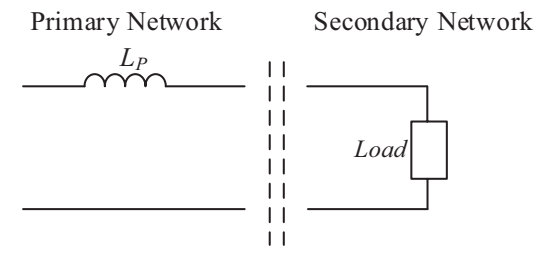

(a)

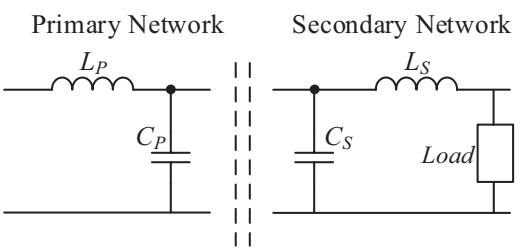

(b)
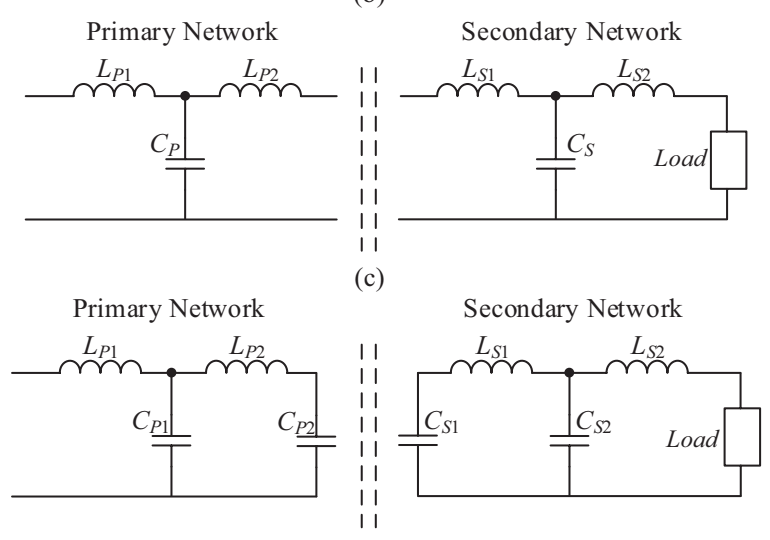

(d)

Fig. 2. Matching networks for capacitive WPT systems: (a) series $L$, (b) doublesided $L C$, (c) double-sided $L C L$, (d) double-sided $L C L C$.

the amount of power transfer of the system, i.e., the lower the coupling capacitance is, proportionally lower the maximum power the system is able to transfer [3].

\section{B. Equivalent Representations of Matching Networks}

Matching L-type structures such as a series inductor combined with a parallel capacitor (series-parallel $L C$, primary in Fig. 2(b)) and a parallel capacitor combined with a series inductor (parallelseries $L C$, secondary in Fig. 2(b)) can be described by a two-port network with gyrator characteristics [26]. A gyrator is a passive, lossless, linear two-port transformation network in which the output and input currents depend on the input and output voltages, respectively, with respect to its trans-conductance gain $G$. In circuit theory, gyrators are often used to reflect inductance using capacitance, impedance into admittance, and vice versa [27]-[30]. The input-output relationship of an ideal gyrator as a two-port network can be expressed as

$$
\left[\begin{array}{l}
I_{1} \\
I_{2}
\end{array}\right]=\left[\begin{array}{cc}
0 & -G \\
G & 0
\end{array}\right]\left[\begin{array}{l}
V_{1} \\
V_{2}
\end{array}\right]
$$

In the context of the above discussion, voltage and current relationships of a series-parallel $L C$ matching structure (Fig. 3(a)) can be derived as follows 


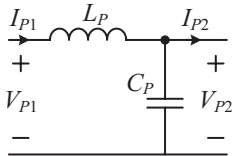

(a)

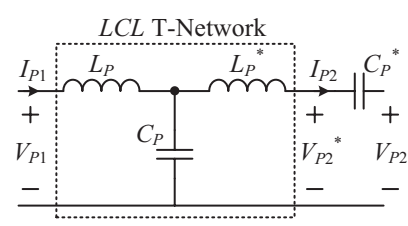

(b)

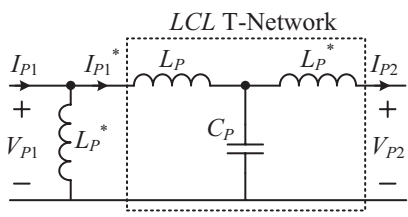

(c)

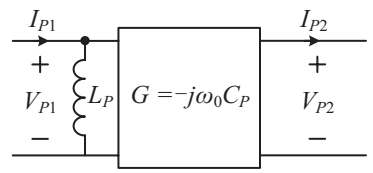

(d)
Fig. 3. Equivalent representations of series-parallel $L C$ matching network. (a) L-type series-parallel resonant $L C$ circuit. (b) Series resonator connected to the L-type circuit for T-type network arrangement. (c) Modified T-type seriesparallel $L C$ resonant circuit. (d) Two-port network with gyrator characteristics.

$$
\left\{\begin{array} { l } 
{ V _ { P 1 } = j \omega L _ { P } I _ { P 1 } + V _ { P 2 } } \\
{ V _ { P 2 } = \frac { 1 } { j \omega C _ { P } } ( I _ { P 1 } - I _ { P 2 } ) }
\end{array} \Rightarrow \left\{\begin{array}{l}
I_{P 1}=\frac{\left(V_{P 1}-V_{P 2}\right)}{j \omega L_{P}} \\
V_{P 1}=j \omega L_{P} I_{P 1}+\frac{1}{j \omega C_{P}}\left(I_{P 1}-I_{P 2}\right)
\end{array}\right.\right.
$$

Assuming operation at the resonant frequency, the currents $I_{P 1}$ and $I_{P 2}$ are given by

$$
\left\{\begin{array}{l}
I_{P 1}=-j \omega_{0} C_{P}\left(V_{P 1}-V_{P 2}\right) \\
I_{P 2}=-j \omega_{0} C_{P} V_{P 1}
\end{array} ; \omega_{0} L_{P}=\frac{1}{\omega_{0} C_{P}},\right.
$$

where $\omega_{0}$ is the resonant angular frequency.

Employing few configurational modifications to the L-type network of Fig. 3(a), an equivalent representation can be obtained. As can be seen in Fig. 3(b), by addition of a series resonator (whereas $L_{P}{ }^{*}=L_{P}$ and $C_{P}{ }^{*}=C_{P}$ ) the network is transformed into a T-type one $\left(L_{P}-C_{P}-L_{P}{ }^{*}\right)$ with an output series capacitance $C_{P}{ }^{*}$. The resultant voltage and current relationships have two-port gyrator characteristics as follows

$$
\begin{aligned}
& V_{P 1}=j \omega_{0} L_{P} I_{P 1}+\frac{1}{j \omega_{0} C_{P}}\left(I_{P 1}-I_{P 2}\right)=\frac{1}{-j \omega_{0} C_{P}} I_{P 2} \\
& V_{P 2}{ }^{*}=-j \omega_{0} L_{P} I_{P 2}+\frac{1}{j \omega_{0} C_{P}}\left(I_{P 1}-I_{P 2}\right)=\frac{1}{j \omega_{0} C_{P}} I_{P 1},
\end{aligned}
$$

and in a matrix representation, (4) can be written as

$$
\left[\begin{array}{l}
I_{P 1} \\
I_{P 2}
\end{array}\right]=\left[\begin{array}{cc}
0 & \underbrace{j \omega_{0} C_{P}}_{G} \\
\underbrace{-j \omega_{0} C_{P}}_{G} & 0
\end{array}\right]\left[\begin{array}{l}
V_{P 1} \\
V_{P 2}^{*}
\end{array}\right],
$$

where the trans-conductance gain is $G=-j \omega_{0} C_{P}$. It should be noted that assuming operation in resonance, the addition of the series branch is an effectively short circuit and does not change the behavior of the circuit.

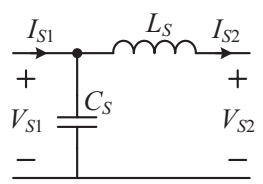

(a)

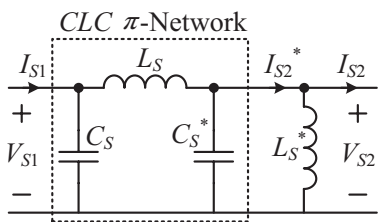

(b)

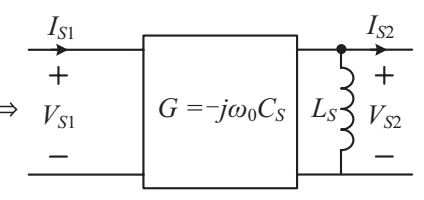

(c)
Fig. 4. Equivalent representations of parallel-series $L C$ matching network. (a) L-type parallel-series resonant $L C$ circuit. (b) Parallel resonator connected to the L-type circuit for $\pi$-type network arrangement. (c) Two-port network with gyrator characteristics.

Following the above observations, the series output capacitance $C_{P}{ }^{*}$ can be reflected to an input parallel inductance $L_{P}{ }^{*}$, as shown in Fig. 3(c). Finally, the original L-type network can be represented as a gyrator element (Fig. 3(d)) with parallel input inductance $L_{p}$.

In analogy to the above practice for the series-parallel $L C$ matching network, the parallel-series $L C$ structure in Fig. 4(a) can also be defined by gyration ratio. This is facilitated by addition of a parallel resonator (whereas ${L_{P}}^{*}=L_{P}$ and $C_{P}{ }^{*}=$ $C_{P}$ ) as shown in Fig. 4(b). Similarly, to the case of the series branch, the parallel resonator is an effectively open circuit when operating at resonance and does not change the original characteristics of the circuit. The L-network is transformed into a $\pi$-type one $\left(C_{S}-L_{S}-C_{S}^{*}\right)$ with parallel output inductor $L_{S}^{*}$. The parallel-series $L C$ structure can be described as a gyrator element with parallel output inductor $L_{S}$ as shown in Fig. 4(c).

\section{Modeling and Analysis of CAPacitive Wireless Power Transfer System}

Following the derivations made in Section II, a capacitivelycoupled power transfer system with double-sided $L C$ matching networks is analyzed. The schematic diagram of the full system is shown in Fig. 5. This loosely-coupled configuration has been selected for the case study demonstration since it can be employed in a variety of medium power level CPT applications [1], [5], [7]. As can be seen in Fig. 5, the matching networks are a series-parallel $L C$ circuit at the primary and a parallel-series $L C$ at the secondary. The capacitive medium is modeled by a $\pi$-network, such that $C_{M}$ is the equivalent mutual capacitance and $C_{M 1}$ and $C_{M 2}$ are the self-capacitances of the coupling plates [4], [5], [7], [31], [32]. The system is driven by a full-bridge inverter on the primary side, and the load is fed via a diode rectifier that is connected to the secondary's network. Due to the structural constraints of the coupling plates, as a general practice, it is assumed that the coupling capacitance $C_{M}$ is significantly lower than the total parallel capacitance. Consequently, the driving frequency is near the matching networks' resonant frequency (i.e., $f_{0}=1 /\left(2 \pi \sqrt{L_{p} C_{p}}\right)=1 /\left(2 \pi \sqrt{L_{s} C_{s}}\right)$. Since high- $Q$ operation is naturally facilitated because of the high 


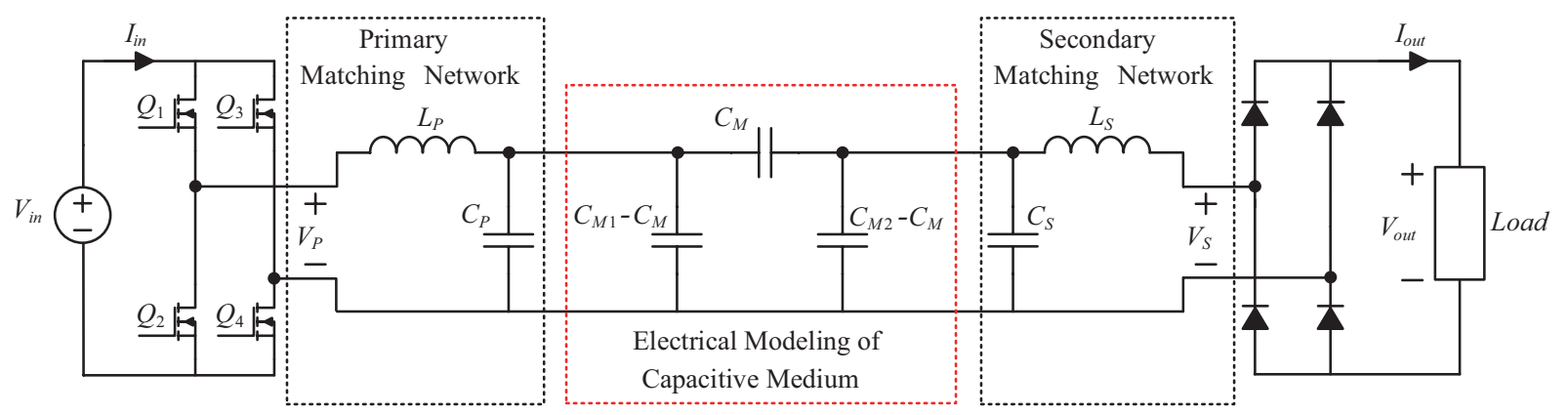

Fig. 5. Schematic diagram of a double-sided $L C$ capacitive WPT system.

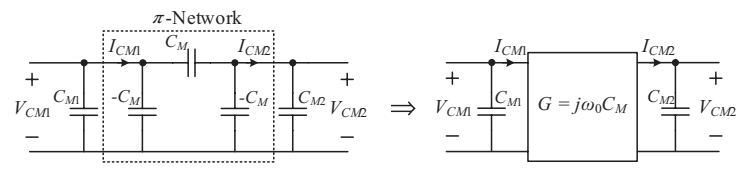

(a)

(b)
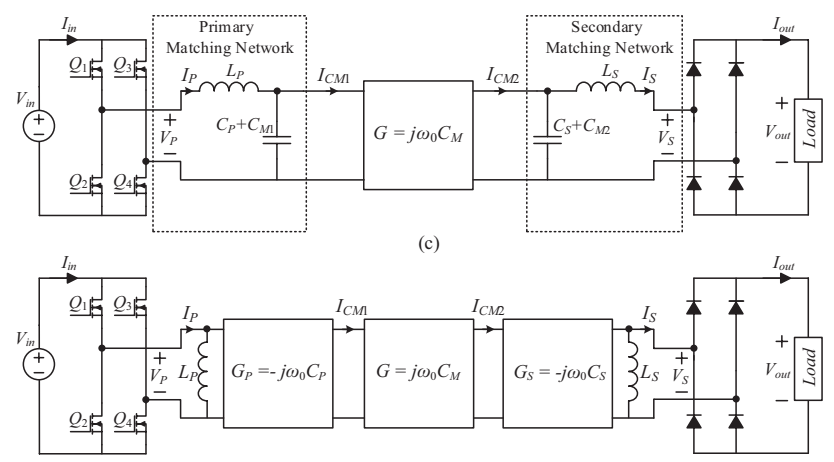

(d)

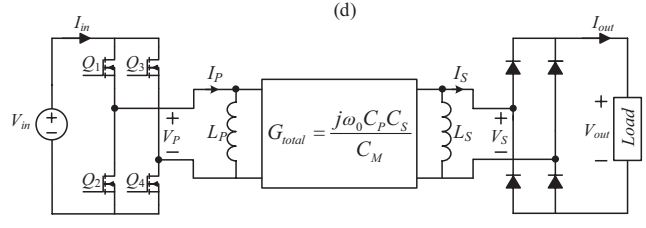

(e)

Fig. 6. Equivalent two-port network models of the analyzed double-sided $L C$ capacitive WPT system. (a) Electrical equivalent model of four-plate capacitive medium. (b) Representation of the capacitive medium with a two-port network. (c) Representation of double-sided $L C$ capacitive WPT system with a gyrator as the capacitive medium. (d) Equivalent circuit of double-sided $L C$ capacitive WPT system with three series gyrators. (e) Equivalent simplified circuit of double-sided $L C$ capacitive WPT system with a single gyrator.

output impedance of the primary's network, the currents as well as voltages of the reactive elements are virtually sinusoidal.

For the derivations of the model, the circuit of Fig. 5 is simplified by separating the parallel capacitances of the medium from the model self-capacitances, as shown in Fig. 6(a). This forms a $\pi$-network constructed by the mutual capacitance $C_{M}$, which can be analyzed similarly to the $C L C \pi$-matching network from Section II. This yields a gyrator element to represent the coupling behavior, as depicted in Fig. 6(b). In the context of the overall system, Fig. 5 is simplified as delineated in Fig. 6(c) (where $C_{P} \gg>C_{M 1}$ and $C_{S} \gg C_{M 2}$ ). Assigning the network dualities that have been established earlier, the entire system is represented by three gyrators connected in series, as shown in Fig. 6(d). This is further reduced to a single gyrator,
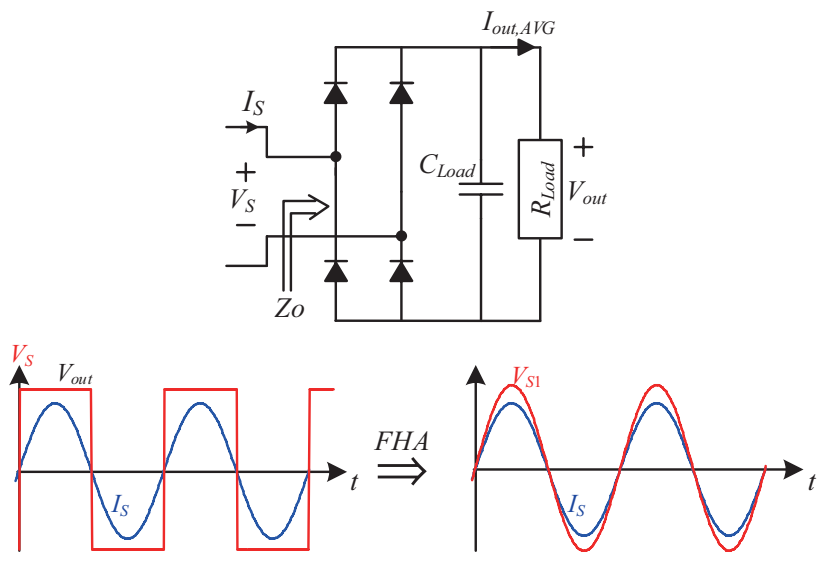

Fig. 7. Secondary side waveforms employing fundamental harmonic approximation.

as illustrated in Fig. 6(e), with total trans-conductance gain that can be expressed as

$$
G_{\text {total }}=\frac{j \omega_{0} C_{P} C_{S}}{C_{M}} .
$$

The simplified circuit of the double-sided $L C$ capacitive WTP system in Fig. 6(e) can now be analyzed as a two-port network with gyrator characteristics, such that the current-voltage relationships are expressed as follows:

(a) $I_{P}=\frac{V_{P}}{j \omega_{0} L_{P}}-\frac{j \omega_{0} C_{P} C_{S}}{C_{M}} V_{S}=-j\left(\frac{V_{P}}{\omega_{0} L_{P}}+\frac{\omega_{0} C_{P} C_{S}}{C_{M}} V_{S}\right)$

(b) $I_{S}=-\frac{V_{S}}{j \omega_{0} L_{S}}+\frac{j \omega_{0} C_{P} C_{S}}{C_{M}} V_{P}=j\left(\frac{V_{S}}{\omega_{0} L_{S}}+\frac{\omega_{0} C_{P} C_{S}}{C_{M}} V_{P}\right)$,

where $I_{P}, V_{P}, I_{S}, V_{S}$ are phasors. Assuming that the phase shift due the diode rectifier is negligible and that the load is a battery or capacitive filter with a long time constant, the current $I_{S}$ and the voltage $V_{S}$ are in phase. Hence, employing the fundamental harmonic approximation [33], the secondary's square wave voltage, $V_{\mathrm{S}}$, can be represented by its fundamental component as depicted in Fig. 7. Therefore, the output of the system can be analyzed in a straightforward manner as follows

$$
\left\{\begin{array}{l}
I_{S}=\frac{\pi}{2} I_{\text {out }, A V G} \\
V_{S}=\frac{\pi}{4} V_{\text {out }}
\end{array} \Rightarrow Z_{O}=\frac{V_{S}}{I_{S}}=\frac{8}{\pi^{2}} R_{\text {Load }},\right.
$$


substituting (8) into (7)-(b), and after some manipulations yields

$$
\begin{aligned}
\frac{V_{S}}{Z_{O}} & =j \frac{V_{S}}{\omega_{0} L_{S}}+j \frac{\omega_{0} C_{P} C_{S}}{C_{M}} V_{P} \\
& \Rightarrow V_{S}\left(\frac{1}{Z_{O}}+\frac{1}{j \omega_{0} L_{S}}\right)=j \frac{\omega_{0} C_{P} C_{S}}{C_{M}} V_{P}
\end{aligned}
$$

from (9), primary-to-secondary voltage ratio can be expressed as follows:

$$
\begin{aligned}
\frac{V_{P}}{V_{S}} & =-\frac{C_{M}}{C_{P}}\left(1+j \frac{\omega_{0} L_{S}}{Z_{O}}\right) \\
& \Rightarrow \angle\left(V_{P}, V_{S}\right)=180^{\circ}-\operatorname{arctg}\left(Q_{S}\right)=90^{\circ}
\end{aligned}
$$

this can be further simplified by defining the secondary's quality factor, $Q_{S}$, as

$$
Q_{S}=\frac{\sqrt{L_{S} / C_{S}}}{Z_{0}}=\frac{\omega_{0} L_{S}}{Z_{O}} \Rightarrow \frac{V_{P}}{V_{S}}=-\frac{C_{M}}{C_{P}}\left(1+j Q_{S}\right) .
$$

By substituting (11) into (7) and rearranging the equations, the current $I_{P}$ can be rewritten as

$$
\begin{aligned}
I_{P} & =-\frac{C_{M}}{C_{S}}\left(\frac{1}{Z_{O}}+j \frac{C_{P} C_{S}-C_{M}^{2}}{\omega_{0} L_{S} C_{M}^{2}}\right) V_{S} \\
& =-C_{M} \omega_{0}\left(Q_{S}+j\left(\frac{1}{k_{C}}-1\right)\right) V_{S},
\end{aligned}
$$

where $k_{C}$ is the capacitive coupling coefficient and is defined as $k_{C}=\left(C_{M} / \sqrt{C_{P} C_{S}}\right)$.

Following the same procedure, the current $I_{s}$ can be also rewritten as

$$
I_{S}=-\frac{C_{P}}{C_{M}} \frac{1}{Z_{0}+j \omega_{0} L_{S}} V_{P}=-\frac{C_{P}}{C_{M} Z_{O}} \frac{1}{1+j Q_{S}} V_{P}
$$

typically, the coupling coefficient $k_{C}$ is relatively small $\left(k_{C}<<1\right)$, and assuming $Q_{S} \gg 1$ the system's current-voltage relationships can be simplified to generic expression as follows:

$$
\left\{\begin{array}{l}
\left|I_{P}\right|=\frac{C_{M} \omega_{0}}{k_{C}^{2}}\left|V_{S}\right|=\frac{\omega_{0} C_{P} C_{S}}{\frac{C_{M}}{G_{\text {total }}}}\left|V_{S}\right| \\
\left|I_{S}\right|=\frac{C_{P}}{C_{M} Z_{O} Q_{S}}\left|V_{P}\right|=\frac{\omega_{0} C_{P} C_{S}}{\frac{C_{M}}{G_{\text {total }}}}\left|V_{P}\right|
\end{array},\right.
$$

it can be well noticed that the double-sided LC CPT system has the characteristics of a pure gyrator, which is in perfect agreement with the analysis given in [1].

The implication of this analysis is that the double-sided $L C$

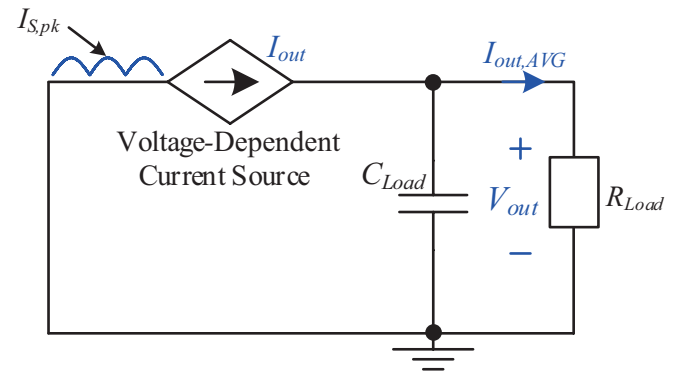

Fig. 8. Behavioral model of the double-sided $L C$ capacitive WPT system for the output side.

capacitive WPT system can be modeled as a voltage dependent current source, $I_{\text {out }}$, which represents the rectified current of $I_{s}$ as illustrated in Fig. 8. Following the above analysis, the average value of the output current $I_{o u t, A V G}$ is found as a function of the trans-conductance gain and the input voltage $V_{\text {in }}$ as follows:

$$
\begin{aligned}
V_{P} & =\frac{4}{\pi} V_{\text {in }} \\
& \Rightarrow I_{\text {out }, A V G}=\frac{8}{\pi^{2}} \frac{\omega_{0} C_{P} C_{S}}{C_{M}} V_{\text {in }},
\end{aligned}
$$

thus, the average output power $P_{\text {out } A V G}$ can be expressed as

$$
P_{\text {out }, A V G}=\left(\frac{8}{\pi} \frac{\omega_{0} C_{P} C_{S}}{C_{M}} V_{\text {in }}\right)^{2} \cdot R_{\text {Load }} .
$$

From (15), it can be observed that for the double-sided $L C$ CPT system the output current (as well as the output power) is inversely proportional to the mutual coupling capacitance $C_{M}$.

\section{Model VALIDATION}

\section{A. Simulation Results}

To verify the behavioral model, a simulation test-bench for the analyzed capacitive WPT system has been constructed in PSIM (PowerSim, Inc.). The input voltage is $30 \mathrm{~V}$ and the medium mutual capacitance $C_{M}=4.5 \mathrm{pF}$, at resonant frequency $f_{0} \approx 1.55 \mathrm{MHz}$ for a load resistance of $R_{\text {Load }}=15 \Omega$ and output capacitor $C_{\text {Load }}=200 \mu \mathrm{F}$. First set of simulations has been carried out for symmetrical matching parameters: $L_{P}=L_{S}=67 \mu \mathrm{H}$, $C_{P}=C_{S}=156 \mathrm{pF}$. The simulation results of the currents and voltages of the primary and secondary sides are shown in Fig. 9(a) and (b), respectively, whereas the dashed lines show the behavioral model predictions. The obtained peak values of the currents are in good agreement with the theoretical predictions in (14), which further implies that the average output current, $I_{\text {out } A V G}$, is as expected from (15).

The second set of simulations has been carried out for asymmetrical matching parameters: $L_{P}=67 \mu \mathrm{H}, C_{P}=156 \mathrm{pF}$ for the primary side, and $L_{S}=90 \mu \mathrm{H}, C_{S}=116 \mathrm{pF}$ for the secondary side. Fig. 10 depicts simulation waveforms of the currents and 


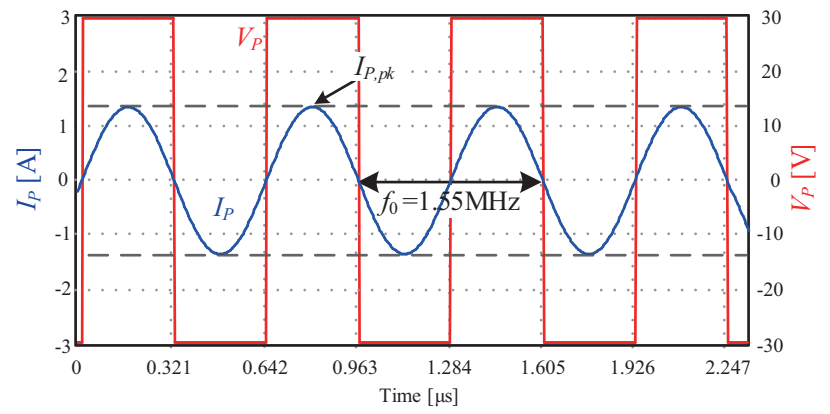

(a)

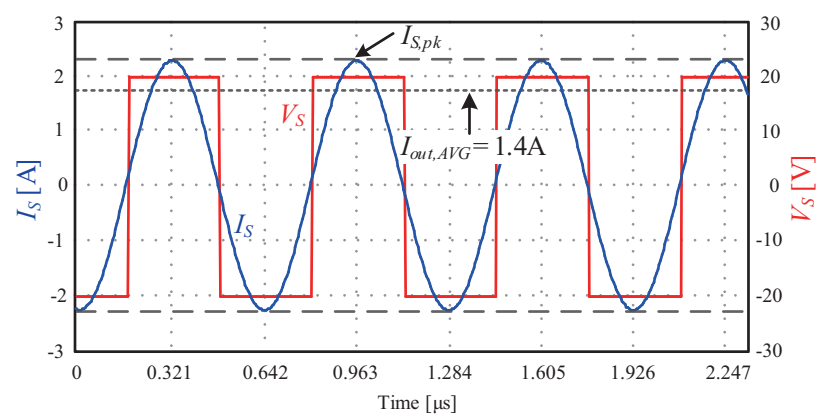

(b)

Fig. 9. Simulated waveforms of the currents and voltages for symmetrical matching parameters (dashed lines are the behavioral model predictions): (a) primary, (b) secondary.

TABLE I

Simulation Test-Benches Values and Parameters

\begin{tabular}{lcc}
\hline \hline Parameter & Fig. 9 & Fig. 10 \\
\hline Input voltage $V_{\text {in }}$ & $30 \mathrm{~V}$ & $30 \mathrm{~V}$ \\
Load resistance & $15 \Omega$ & $15 \Omega$ \\
Output capacitor & $200 \mu \mathrm{F}$ & $200 \mu \mathrm{F}$ \\
$C_{M}$ & $4.5 \mathrm{pF}$ & $4.5 \mathrm{pF}$ \\
Inductors $L_{P}$ and $L_{S}$ & $67 \mu \mathrm{H}$ & $67 \mu \mathrm{H}, 90 \mu \mathrm{H}$ \\
Capacitors $C_{P}$ and $C_{S}$ & $156 \mathrm{pF}$ & $156 \mathrm{pF}, 116 \mathrm{pF}$ \\
Resonant frequency $f_{0}$ & $1.55 \mathrm{MHz}$ & $1.55 \mathrm{MHz}$ \\
$I_{\text {out }, A V G}$ & $1.4 \mathrm{~A}$ & $1.1 \mathrm{~A}$ \\
\hline \hline
\end{tabular}

voltages of the primary and secondary. As can be seen, the obtained results match the model predictions. It can also be noticed that the resulting output current is lower than that in the symmetrical case shown in Fig. 9. This is due to the lower trans-conductance gain in the case with a lower value of $C_{S}$. Table I summarizes the operating conditions and parameters for Figs. 9 and 10.

In addition, the calculated results for the average output current of the asymmetrical case are plotted against the results obtained from the simulation. These are depicted in Fig. 11, where Fig. 11(a) shows the current as a function of the input voltage, and Fig. 11(b) shows the current as a function of the coupling capacitances. A very good agreement is obtained throughout these simulations. As can be seen in Fig. 11(b), as predicted by the model in (15), the output current is inversely proportional to the mutual coupling capacitance $C_{M}$. Therefore, the output power will follow a similar trend.

In order to evaluate and predict the performance of a double-

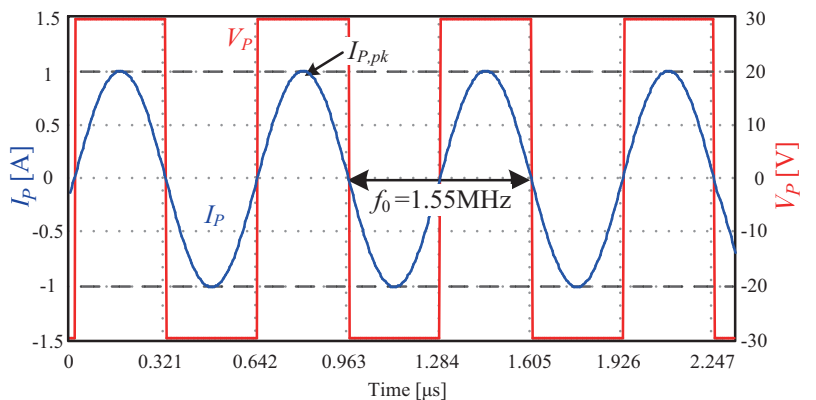

(a)

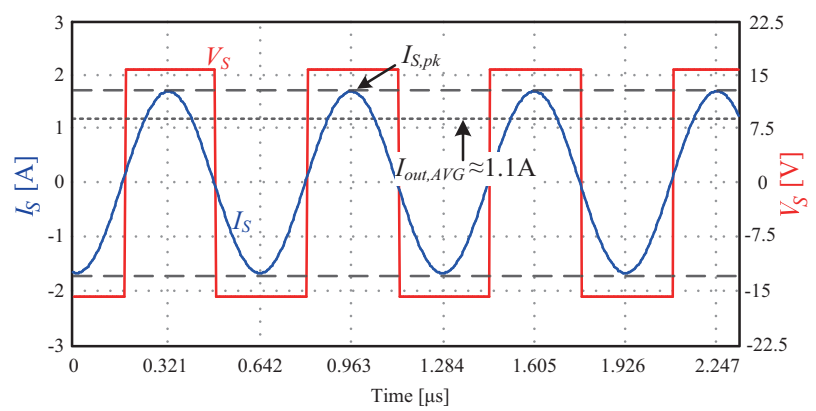

(b)

Fig. 10. Currents and voltages simulation results for asymmetrical matching parameters (dashed lines are the behavioral model predictions): (a) primary, (b) secondary.

sided $L C$ system for medium variations (distance/misalignment), a simulation test-bench with variable capacitors has been constructed as shown in Fig. 12. There, for convenience, the capacitive medium is illustrated by variable capacitor symbols. The methodology to model a continuous-time, variable inductance as described previously in [34], [35] has been employed and adapted to describe varying capacitance in this study (detailed implementation of continuous-time, variable, capacitor is beyond the scope of this paper and are provided subsequent publication).

Simulations have been carried out under nominal operating conditions and matching networks that are identical to those of the asymmetrical case study, whereas the initial coupling capacitance is 5 pF. Fig. 13(a) shows the result of the average value of $I_{\text {out }}$ for a $2 \mathrm{pF}$ variation of the mutual capacitance, such that the final coupling capacitance is $C_{M}=7 \mathrm{pF}$. The solid red line shows the simulation result, whereas the dashed blue markers show the behavioral model outcome. It can be observed that the results of the cycle-by-cycle simulation and behavioral model are in very good agreement. It can be further noticed that when $C_{M}$ increases, the output current decreases as expected from the theoretical analysis. Fig. 13(b) shows the output current for a medium step-down variation of $2 \mathrm{pF}$, such that the final coupling capacitance is $C_{M}=3 \mathrm{pF}$. The output current settles on approximately $1.7 \mathrm{~A}$ for both the model and cycle-bycycle simulation, which suits well the theoretical predictions as shown in detail in Fig. 11(b).

\section{B. Capacitive Coupler Design}

To facilitate reliable estimation of the capacitive coupler 


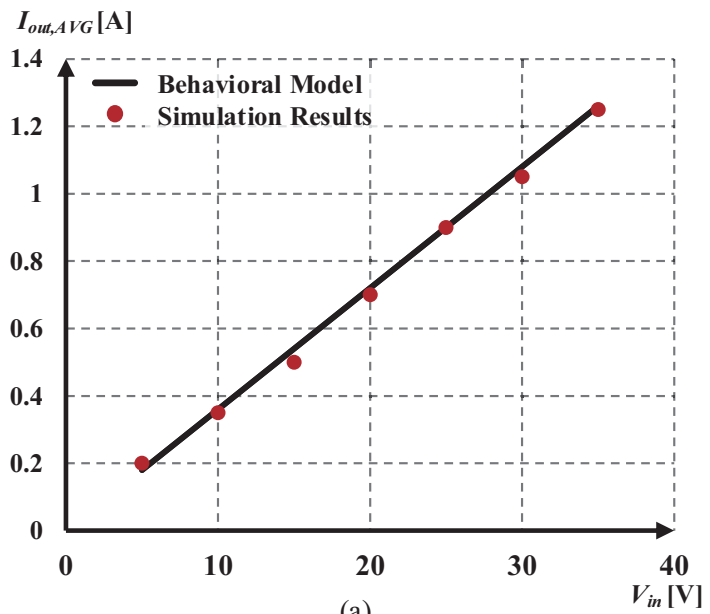

(a)

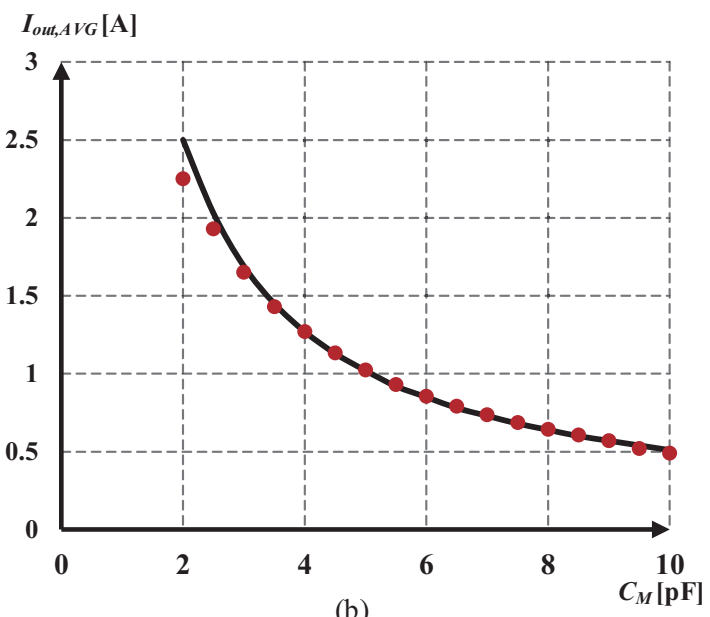

(b)

Fig. 11. Behavioral model and simulated average output current, $I_{\text {out.AVG }}$, curves an asymmetrical setup. (a) As a function of the input voltage $V_{i n}$. (b) As a function of the mutual coupling capacitance $C_{M}$.

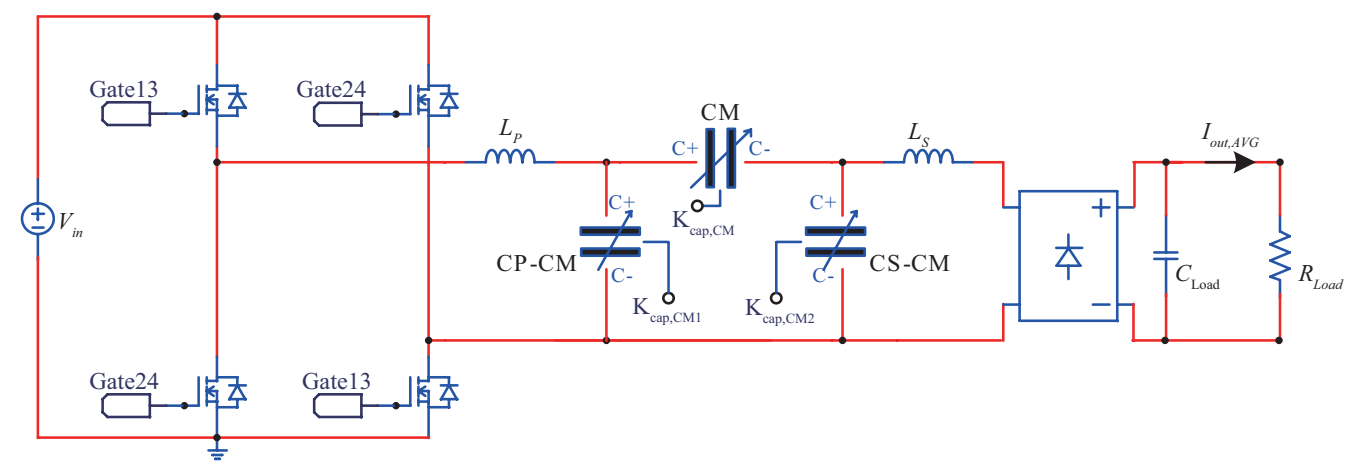

Fig. 12. Schematic simulation test-bench of the WPT system with variable capacitor symbol illustrations for the capacitive medium.

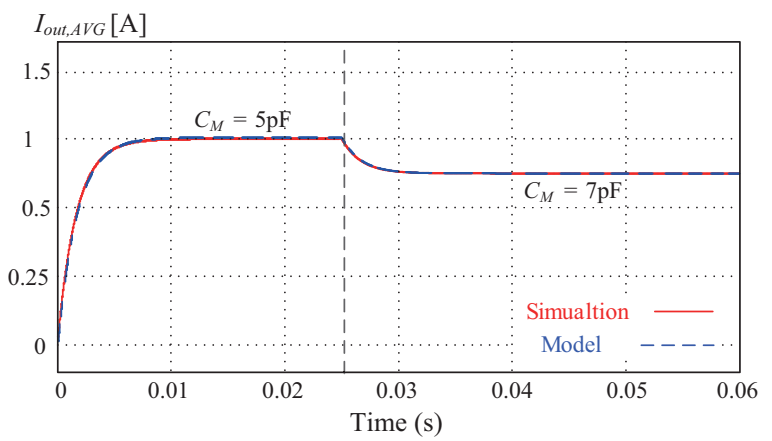

(a)

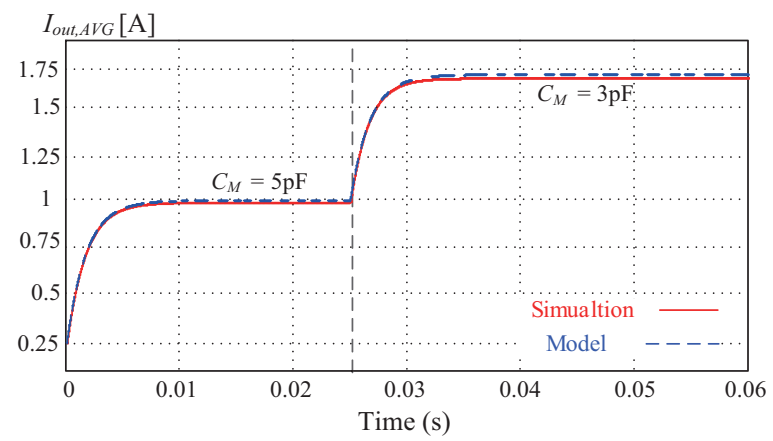

(b)

Fig. 13. Average output current for capacitive medium variations with initial mutual capacitance $C_{M}=5 \mathrm{pF}$. (a) Step-up of $2 \mathrm{pF}$. (b) Step-down of $2 \mathrm{pF}$.

for the experimental measurements, estimation of the plates capacitances has been carried out by Maxwell (Ansys) Finite Element Analysis (FEA) tool (Fig. 14(a)). Rigorous simulation procedure over various air-gaps has been carried out to determine the mutual capacitance, $C_{M}$, for the symmetrical copperbased capacitive coupler, whereas each plate is $170 \mathrm{~mm} \times 170 \mathrm{~mm}$. The results for the mutual coupling capacitance, $C_{M}$, and for the coupling coefficient, $k_{C}$, are shown in Fig. 14(b) and (c), respectively. It can be observed that the coupling coefficient is
$<<1$ over the entire range, while $C_{M}$ decreases by nearly four times at an air-gap of $70 \mathrm{~mm}$.

It should be noted that typically, FEA are generated by defining the geometry of the element and by setting the boundary conditions, in the context of the capacitive coupler these are four symmetrical copper plates and voltage excitations to the plates. The mutual capacitances of the coupling plates have been calculated based on the equations in [4], [5] and the coupling coefficient, $k_{C}$, has been calculated by $C_{M} / \sqrt{C_{P} C_{S}}$. 


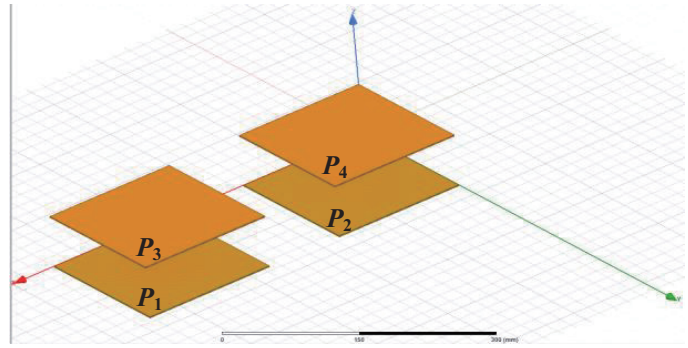

(a)

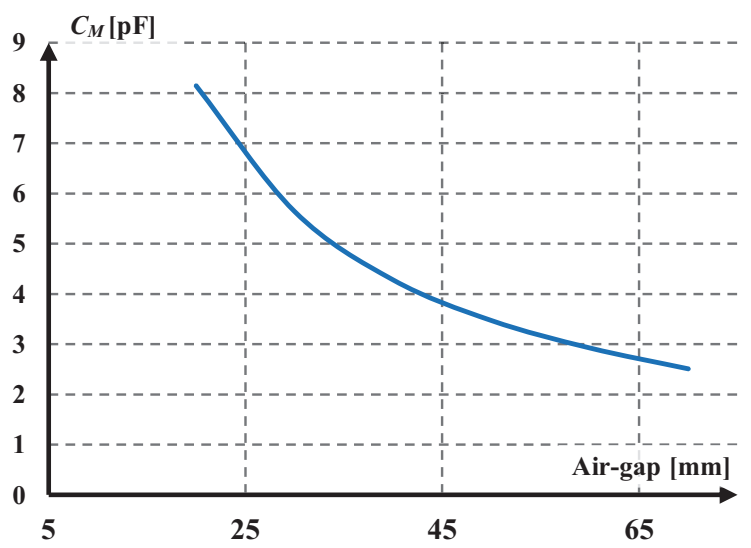

(b)

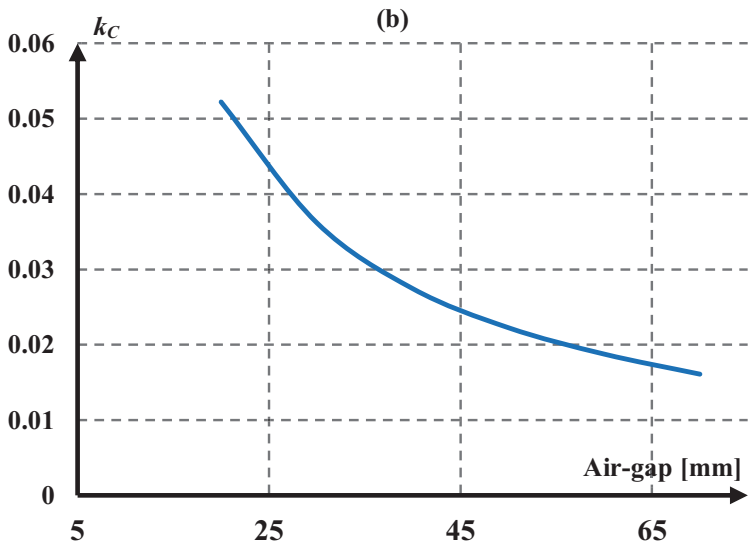

(c)

Fig. 14. Maxwell (Ansys software) simulation test-bench for the capacitive coupler design.

\section{EXPERIMENTAL VERIFICATION}

Based on the above analysis and simulations an experimental double-sided $L C$ capacitive WPT prototype (Fig. 15), has been designed and examined. Since the coupling plates have been designed symmetrically, the matching networks have been also designed to be symmetrical, with $L_{P}=L_{S} \approx 67 \mu \mathrm{H}$ and $C_{P}=C_{S}=156 \mathrm{pF}$. The gate drive signals of the full-bridge inverter were generated with a Cyclone IV FPGA [36] at an operating frequency slightly above the resonance $f_{0} \approx 1.558 \mathrm{MHz}$ guaranteeing soft-switching operation. The full-bridge inverter has been implemented with GaN modules operable at several $\mathrm{MHz}$ [37]. To reduce the limitations due to magnetic and skin-effect losses in the $\mathrm{MHz}$ range [38], [39], the matching inductors $L_{p}$ and $L_{s}$ have been constructed with AWG 48 litz

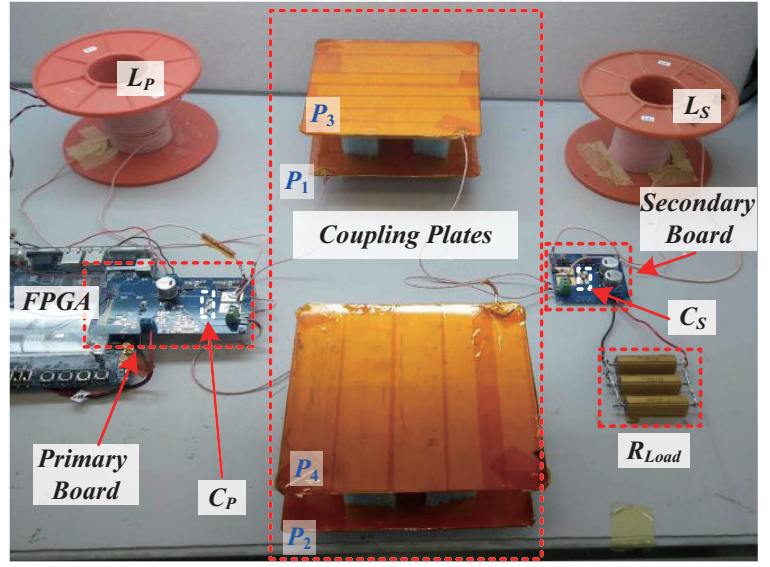

Fig. 15. Experimental setup of a capacitive WPT prototype.

TABLE II

Experimental Prototype Values and Parameters

\begin{tabular}{lc}
\hline \hline Parameter & Value/Type \\
\hline Input voltage $V_{\text {in }}$ & $30 \mathrm{~V}$ \\
$I_{\text {out }, A V G}$ & up to $1.6 \mathrm{~A}$ \\
Load resistance $R_{\text {Load }}$ & $\sim 15 \Omega$ \\
Coupling plates & $170 \mathrm{~mm} \times 170 \mathrm{~mm}$ \\
$C_{M} /$ air-gaps & $2.5 \mathrm{pF}-8 \mathrm{pF} / 20 \mathrm{~mm}-70 \mathrm{~mm}$ \\
Full-bridge transistors & $\mathrm{LMG} 5200,80 \mathrm{~V}, 15 \mathrm{~m} \Omega, \mathrm{Dual}$ \\
Rectifier diodes & $5 \mathrm{~A} / 200 \mathrm{~V}, \mathrm{VSSC} 520 \mathrm{~S}-\mathrm{M} 3$ \\
Inductors $L_{P}$ and $L_{S}$ & $\sim 67 \mu \mathrm{H}$ \\
Capacitors $C_{P}$ and $C_{S}$ & $156 \mathrm{pF}, \mathrm{AVX} \mathrm{MLLC} 5 \mathrm{kV}$ \\
Output capacitor $C_{\text {Load }}$ & $200 \mu \mathrm{F}, \mathrm{EEV}-\mathrm{FK} 2 \mathrm{~A} 101 \mathrm{M}$ \\
Resonant frequency $f_{0}$ & $\sim 1.558 \mathrm{MHz}$ \\
\hline
\end{tabular}

wire wrapped on an air-core. High-voltage multilayer SMD ceramic capacitors have been used in parallel to form the desired matching capacitors $C_{P}$ and $C_{S}$. The overall operating conditions and parameters of the experimental prototype are summarized in Table II.

Fig. 16 shows experimental waveforms of the system for an air-gap of $30 \mathrm{~mm}$ (corresponds to $C_{M} \approx 4.5 \mathrm{pF}$ approximately), whereas the input voltage $V_{i n}=30 \mathrm{~V}$ and the load resistance $R_{\text {Load }} \approx 15 \Omega$. Fig. 16(a) shows the primary waveforms, as can be seen $V_{P}$ toggles between $-30 \mathrm{~V}$ to $30 \mathrm{~V}$ and the peak value of the sinusoidal current $I_{P}$ is $\sim 1.22 \mathrm{~A}$. The current at the secondary, $I_{s}$, is shown in Fig. 16(b), taking into consideration that some losses are introduced throughout the experiments, the obtained peak value $(1.8 \mathrm{~A})$ as well as the resulting average value $(\sim 1.1 \mathrm{~A})$ are in the vicinity of the simulation results shown in Section IV, and the system efficiency is $75 \%$. Fig. 17 shows experimental waveforms for an air-gap of $60 \mathrm{~mm}$ which corresponds to $C_{M}$ of $3 \mathrm{pF}$ approximately, again, the measured output voltage and current are well predicted by both the behavioral model and simulations.

To further verify the strength of the behavioral model, the average output current has been measured for various input voltages, for a constant air-gap of $30 \mathrm{~mm}$, the results are summarized in Fig. 18(a). The experimental measurements tightly follow the results obtained by the simulations as well as the model predictions. It can be noticed that the output current 


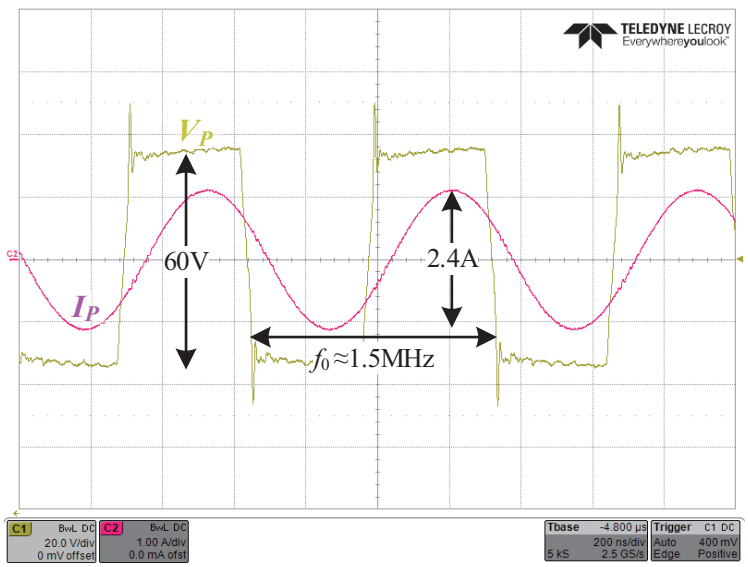

(a)

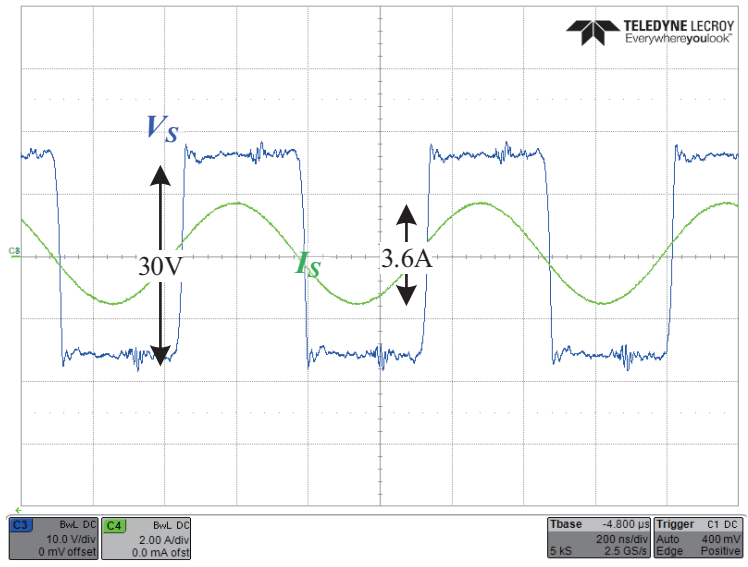

(b)

Fig. 16. Experimental waveforms with operating conditions: $V_{\text {in }}=30 \mathrm{~V}, R_{\text {Load }}=15 \Omega$, coupling capacitance $C_{M} \approx 4.5 \mathrm{pF}$. (a) Primary side $V_{p}: 20 \mathrm{~V} / \mathrm{div}, I_{P}: 1 \mathrm{~A} / \mathrm{div}$, (b) Secondary side $V_{S}: 10 \mathrm{~V} / \mathrm{div}, I_{S}: 2 \mathrm{~A} /$ div, Time scale: $200 \mathrm{~ns} / \mathrm{div}$.

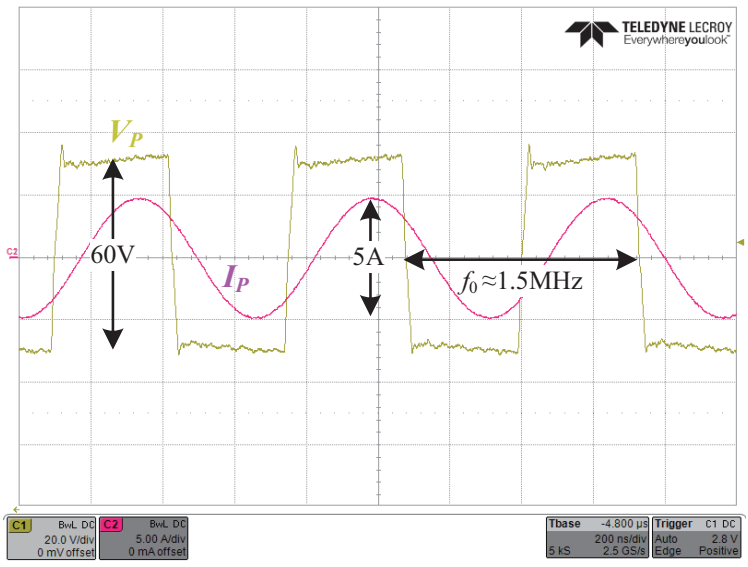

(a)

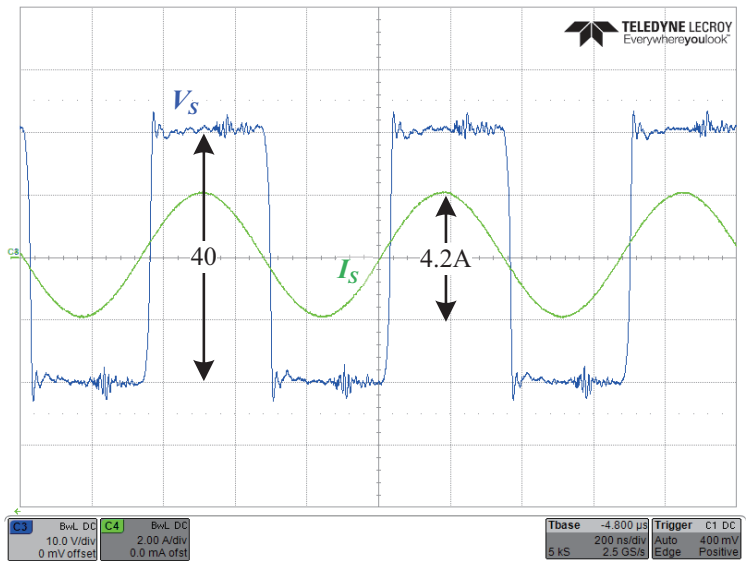

(b)

Fig. 17. Experimental waveforms with conditions: $V_{\text {in }}=30 \mathrm{~V}, R_{\text {Load }}=15 \Omega$, coupling capacitance $C_{M} \approx 3 \mathrm{pF}$, (a)Primary side $V_{P}: 20 \mathrm{~V} / \mathrm{div}, I_{P}: 5 \mathrm{~A} / \mathrm{div}$, (b) Secondary side $V_{S}: 10 \mathrm{~V} / \mathrm{div}, I_{S}: 2 \mathrm{~A} / \mathrm{div}$. Time scale: $200 \mathrm{~ns} / \mathrm{div}$.
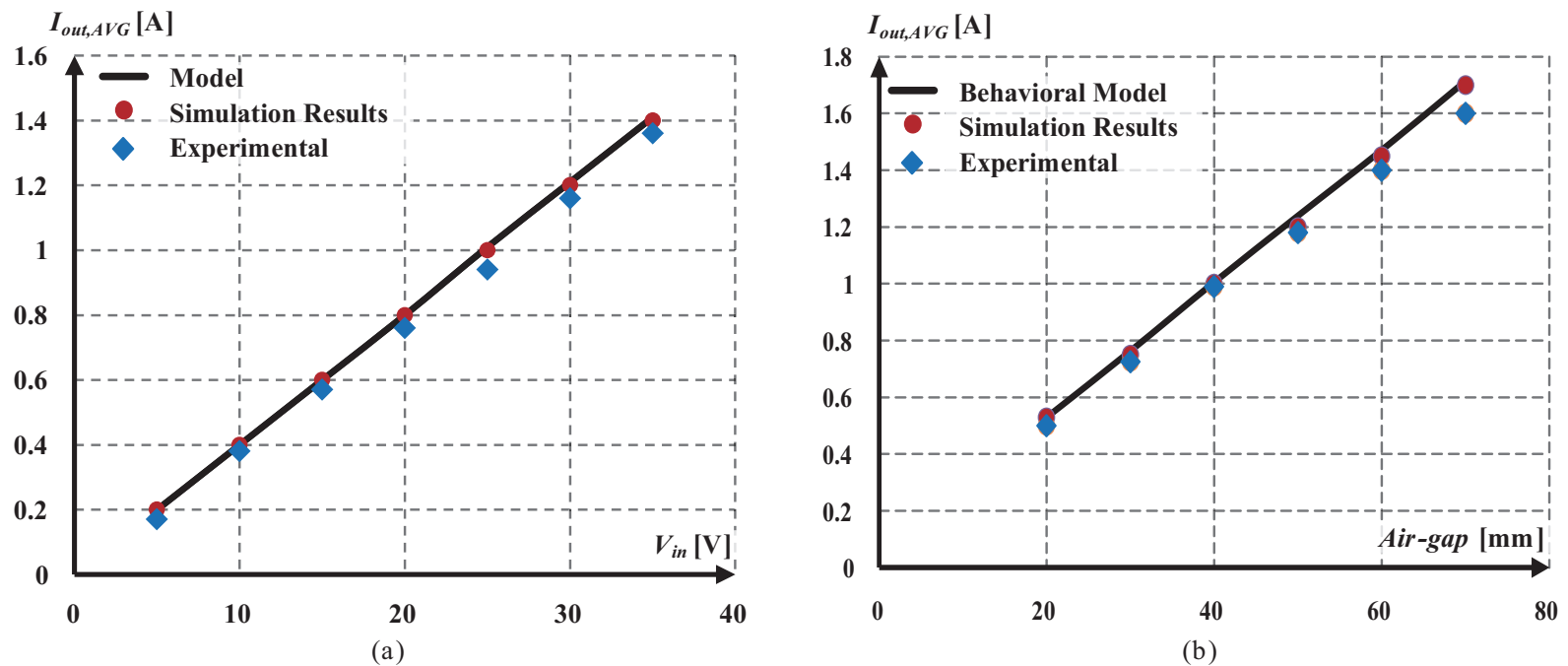

(b)

Fig. 18. Average output current, $I_{\text {out } A V G}$, curves: (a) as a function of the input voltage $V_{\text {in }}$, for $30 \mathrm{~mm}$ air-gap; (b) as a function of the air-gaps (coupling capacitance $C_{M}$ ), for $V_{\text {in }}=30 \mathrm{~V}$. 
(as well as the output power) increases with the input voltage, as predicted from the gyrator current-voltage relationships obtained in Section III. Fig. 18(b) depicts the output current comparison between the experimental, simulated and behavioral model for various air-gaps between $20 \mathrm{~mm}$ and $70 \mathrm{~mm}$, thus demonstrating variations in distance/misalignment (while $V_{\text {in }}=$ $30 \mathrm{~V}$ ). This way, the inversely proportional behavior between the capacitive coupling and the output current is well validated by the experimental measurements.

\section{Conclusions}

A two-port network-based modeling approach for capacitive WPT systems has been presented. Based on the modeling approach, the behavior of resonant-operating capacitive WPT system (or in the vicinity of resonant operation) can be analyzed and described for different system variations such as capacitive coupling interface, resonant frequency and matching networks components. The modeling method provides an insight to the effects of the parameters on the system behavior, the crosscoupling relationships between transmitting and receiving sides, and sourcing features based on the operation mode and settings. The simplicity and generality of the gyrator model as an energy transfer element offers an efficient closed-form alternative to complex analytical approaches or tedious numerical simulations. An experimental CPT prototype operating in the $\mathrm{MHz}$ range has been constructed. The prototype has been evaluated for various air-gaps up to $70 \mathrm{~mm}$ and $45 \mathrm{~W}$ output power delivery. The experimental case-study confirmed the theoretical predictions of the model with an excellent agreement between the analytical derivations, simulations, and experimental results.

\section{ACKNOWLEDGMENT}

This research was supported by the Prof. A. Pazi Research Foundation.

\section{REFERENCES}

[1] E. Abramov and M. M. Peretz, "Modeling and analysis of capacitive wireless power transfer systems: a network approach", in Proc. IEEE Int. Power Electron. Appli. Conf. Expo. (PEAC), Nov. 2018, pp. 1-6.

[2] M. P. Theodoridis, "Effective capacitive power transfer," IEEE Trans. Power Electron., vol. 27, no. 12, pp. 4906-4913, Dec. 2012.

[3] F. Lu, H. Zhang, H. Hofmann, and C. Mi, "A double-sided LCLC compensated capacitive power transfer system for electric vehicle charging," IEEE Trans. Power Electron., vol. 30, no. 11, pp. 6011-6014, Jun. 2015 .

[4] H. Zhang, F. Lu, H. Hofmann, W. Liu, and C. C. Mi, "A four-plate compact capacitive coupler design and LCL-compensated topology for capacitive power transfer in electric vehicle charging application," IEEE Trans. Power Electron., vol. 31, no. 12, pp. 8541-8551, Dec. 2016.

[5] F. Lu, H. Zhang, H. Hofmann, C. Mi, "A loosely coupled capacitive power transfer system with LC compensation circuit topology," in Proc. IEEE Energy Convers. Congr. Expo. (ECCE), pp. 1-5, 2016.

[6] F. Lu, H. Zhang, C. Mi, "A two-plate capacitive wireless power transfer system for electric vehicle charging applications," IEEE Trans. Power Electron, vol. 33, no. 2, pp. 946-969, Aug. 2017.

[7] F. Lu, H. Zhang, H. Hofmann, and C. Mi, "A double-sided LC compensation circuit for loosely-coupled capacitive power transfer," IEEE Trans. Power Electron., vol. 33, no. 2, pp. 1633-1643, Feb. 2017.

[8] T. Imura and Y. Hori, "Maximizing air gap and efficiency of magnetic resonant coupling for wireless power transfer using equivalent circuit and Neumann formula," IEEE Trans. Ind. Electron., vol. 58, no. 10, pp. 4746-4752, Oct. 2011.

[9] J. Sallan, J. L. Villa, A. Llombart, and J. F. Sanz, "Optimal design of ICPT systems applied to electric vehicle battery charge," IEEE Trans. Ind. Electron., vol. 56, no. 6, pp. 2140-2149, Jun. 2009.

[10] S. Jaegue et al., "Design and implementation of shaped magnetic resonance-based wireless power transfer system for roadway-powered moving electric vehicles," IEEE Trans. Ind. Electron., vol. 61, no. 3, pp. 1179-1192, Mar. 2014.

[11] K. Wu, D. Choudhury, and H. Matsumoto, "Wireless power transmission, technology, and applications" in Proc. IEEE, Vol. 101, no.6, pp. 12711275, June 2013.

[12] L. Collins, "Cut the cord," IET Journals \& Magazines., vol. 5, no. 6, pp 42-46, Jan.-Dec. 2007.

[13] F. Lu, H. Zhang, H. Hofmann, C. Mi, "An inductive and capacitive integrated coupler and its LCL compensation circuit design for wireless power transfer," IEEE Trans. Ind. Appl., vol. 53, no. 5, pp. 4903-4913, Oct. 2017.

[14] Y. Lim, H. Tang, S. Lim, J. Park, "An adaptive impedance-matching network based on a novel capacitor matrix for wireless power transfer," IEEE Trans. Power Electron., vol. 29, no. 8, pp. 4403-4413, August 2014.

[15] T.C. Beh, M. Kato, T. Imura, S. Oh and Y. Hori, "Automated impedance matching system for robust wireless power transfer via magnetic resonance coupling," IEEE Trans. Ind. Electron., vol. 60, no. 9, pp. 3689 3698, September 2013.

[16] W. Zhang and C. Mi, "Compensation topologies for high power wireless power transfer systems," IEEE Trans. Veh. Technol., vol. 65, no.6, pp. 4768-4778, July 2015.

[17] A. Kumar, S. Sinha, A. Sepahvand, K. K. Afridi, "Improved design optimization for high-efficiency matching networks," IEEE Trans. Power Electron., vol. 33, no. 1, pp. 37-50, Jan. 2018.

[18] A. Witulski and R. W. Erickson, "Extension of state-space averaging to resonant switches and beyond," IEEE Trans. Power Electron., vol. 5, no. 1, pp. 98-109, Jan. 1990.

[19] H. Hao, G. A. Covic, and J. T. Boys, "An approximate dynamic model of LCL-T-based inductive power transfer power supplies," IEEE Trans. Power Electron., vol. 29, no. 10, pp. 5554-5567, Oct. 2014.

[20] V. Vorperian, R. Tymerski, and F. C. Lee, "Equivalent circuit models for resonant and PWM switches," IEEE Trans. Power Electron., vol. 4, no. 2 , pp. 205-214, Apr. 1989.

[21] D. Maksimovic and S. Cuk, "A unified analysis of PWM converters in discontinuous modes," IEEE Trans. Power Electron., vol. 6, no. 3, pp. 476-490, Jul. 1991.

[22] J. Lu, A. Kumar, K. K. Afridi, "A step-superposition based analysis approach to modeling resonant converters," IEEE Trans. Power Electron., vol. 33, no. 8, pp. 7148-7165, Aug. 2018.

[23] C. J. Anderson and J. A. Lyle, "Technique for evaluating system performance using $\mathrm{Q}$ in numerical simulation exhibiting inter symbol interference," Electron. Lett., vol. 30, pp. 71-72, 1994.

[24] L. Chen, S. Liu, Y. Zhou, and T. Cui, "An optimizable circuit structure for high-efficiency wireless power transfer," IEEE Trans. Ind. Electron., vol. 60, no. 1, pp. 339-349, Jan. 2013.

[25] B. Wang, W. Yerazunis, and K. H. Teo, "Wireless power transfer: Metamaterials and array of coupled resonators," in Proc. IEEE, vol. 101, no. 6, pp. 1359-1368, Jun. 2013.

[26] Y. H. Sohn, B. H. Choi, G. H. Cho, and C. T. Rim, "Gyrator-based analysis of resonant circuits in inductive power transfer systems," IEEE Trans. Power Electron, vol. 31, no. 10, pp. 6824-6843, Oct. 2016.

[27] A. Cid-Pastor, L.Martinez-Salamero, C. Alonso, R. Leyva, and S. Singer, "Paralleling DC-DC switching converters by means of power gyrators," IEEE Trans. Power Electron., vol. 22, no. 6, pp. 2444-2453, Nov. 2007.

[28] D. C. Hamill, "Lumped equivalent circuits of magnetic components: The gyrator-capacitor approach," IEEE Trans. Power Electron., vol. 8, no. 2, pp. 97-103, Apr. 1993.

[29] S. Singer, "Loss-free gyrator realization," IEEE Trans. Circuits and Syst, vol. 35, no. 1, pp. 26-34, Jan. 1988.

[30] S. Singer, "Gyrators application in power processing circuits," IEEE Trans. Ind. Electron., vol. IE-34, no. 3, pp. 313-318, Aug. 1987.

[31] C. Liu, A.P. Hu, and M. Budhia, "A generalized coupling model for capacitive power transfer systems," in Proc. 36th Annu. Conf. IEEE Ind. 
Electron. Society, Glendale, AZ, pp.274-279, Nov. 2010.

[32] L. Huang, A. P. Hu, A. K. Swain, and Y. Su, "Accurate steady-state modeling of capacitive-coupling interface of capacitive power transfer systems with cross-coupling," Wireless Power Transfer, vol. 3, no. 1, pp. 53-62, 2016.

[33] R.L. Steigerwald, "A comparison of half-bridge resonant converter topologies," IEEE Trans. Power Electron., vol. 3, no. 2, pp. 174-182, Apr. 1988.

[34] S. Cheon, Y. H. Kim, S. Y. Kang, M. L. Lee, J. M. Lee, and T. Zyung, "Circuit-model-based analysis of a wireless energy-transfer system via coupled magnetic resonances," IEEE Trans. Ind. Electron., vol. 58, no. 7, pp. 2906-2914, Jul. 2011.

[35] S. Ben-Yaakov and M.M. Peretz, "Simulation bits: A SPICE behavioral model of non-linear inductors," IEEE Power Electron. Soc. Newslett., vol. 15, no. 4, pp. 9-10, 4th Quarter 2003.

[36] DE2 Development and Education Board User Manual, Altera Corporation, 2006.

[37] Texas Instrument: 'LMG5200 80-V, 10-A GaN Half-Bridge Power Stage' [Online]. Available: http://www.ti.com/lit/ds/symlink/lmg5200.pdf, accessed March 2017.

[38] J. Craninckx and M. Steyaert, "A 1.8-GHz low-phase-noise CMOS VCO using optimized hollow spiral inductors," IEEE J. Solid-State Circuits, vol. 32, np. 5, pp. 736-744, May 1997.

[39] M. Bartoli, N. Noferi, A. Reatti, and M. Kazimierczuk, "Modeling litzwire winding losses in high-frequency power inductors," in Proc. IEEE Power Electroni. Specialists Conf., Jun. 23-27, 1996, pp. 1690 1696.

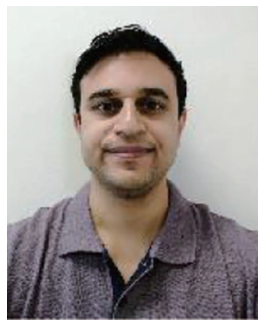

Eli Abramov received the B.Sc. degree in Electrical and Electronics Engineering from the Samy Shamoon College of Engineering, Beer-Sheva, Israel, in 2013, and the M.Sc degree in electrical and computer engineering from Ben-Gurion University, Israel in 2016. He is currently working toward the Ph.D. degree in electrical and computer engineering at the Center for Power Electronics and Mixed-Signal IC, at Ben-Gurion University of the Negev, Israel.

His research interests include power and analog IC, mixed-signal integration for power management systems, power systems on-chip, modeling and control of wireless power transfer systems.

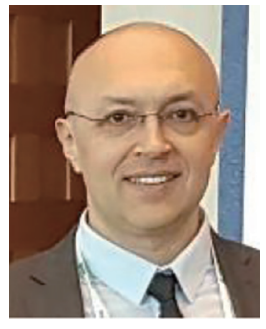

Ilya Zeltser received the B.Sc., M.Sc. and Ph. D. degrees in Electrical and Computer Engineering at the Ben-Gurion University of the Negev, Beer Sheva, Israel, in the years 1995, 1998 and 2010 respectively.

He was a co-founder of Green Power Technologies Ltd., Israel, where he served as VP R\&D during the years 2000-2006. Since 2010, his research activities are being conducted at Rafael Advanced Defense systems Ltd., Israel, where he also held several managerial positions. He currently serves as a Divisional System Engineer.

His current research interests include dc-dc converters, chargers, pulsed power modulators, modeling, renewable energy and wireless power transfer systems.

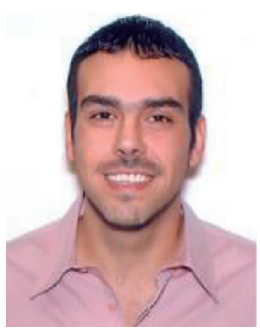

Mor Mordechai Peretz was born in Beer-Sheva, Israel, in 1979. He received the B.Tech. degree in Electrical Engineering from the Negev Academic College of Engineering, Beer-Sheva, in 2003 and the M.Sc. and Ph.D. degrees in electrical and computer engineering from Ben-Gurion University, Negev, Israel, in 2005 and 2010, respectively.

From 2010 to 2012, he was a Postdoctoral Fellow at the Laboratory for Power Management and Integrated SMPS, University of Toronto, Canada. In 2012, he joined the Department of Electrical and Computer Engineering, Ben-Gurion University, where he is currently the director of the Center for Power Electronics and Mixed-Signal IC. Prof. Peretz serves as an associate editor of the IEEE Transactions on Power Electronics and the IEEE Journal of Emerging and Selected Topics in Power Electronics.

His research interests include digital and smart control methods for efficient energy processing, SMPS miniaturization, mixed-signal IC design of SMPS, modeling and computer aided design, applications of nonlinear magnetics and renewable energy systems. 